\title{
Control of pyrethroid and DDT-resistant Anopheles gambiae by application of indoor residual spraying or mosquito nets treated with a long-lasting organophosphate insecticide, chlorpyrifos-methyl
}

\author{
Raphael N'Guessan ${ }^{1,2^{*}}$, Pelagie Boko ${ }^{2}$, Abibathou Odjo ${ }^{2}$, Joseph Chabi ${ }^{2}$, Martin Akogbeto ${ }^{2}$, Mark Rowland ${ }^{1}$
}

\begin{abstract}
Background: Scaling up of long-lasting insecticidal nets (LLINs) and indoor residual spraying (IRS) with support from the Global Fund and President's Malaria Initiative is providing increased opportunities for malaria control in Africa. The most cost-effective and longest-lasting residual insecticide DDT is also the most environmentally persistent. Alternative residual insecticides exist, but are too short-lived or too expensive to sustain. Dow Agrosciences have developed a microencapsulated formulation (CS) of the organophosphate chlorpyrifos methyl as a cost-effective, long-lasting alternative to DDT.
\end{abstract}

Methods: Chlorpyrifos methyl CS was tested as an IRS or ITN treatment in experimental huts in an area of Benin where Anopheles gambiae and Culex quinquefasiactus are resistant to pyrethroids, but susceptible to organophosphates. Efficacy and residual activity was compared to that of DDT and the pyrethroid lambdacyalothrin.

Results: IRS with chlorpyrifos methyl killed 95\% of An. gambiae that entered the hut as compared to $31 \%$ with lambdacyhalothrin and 50\% with DDT. Control of CX. quinquefasciatus showed a similar trend; although the level of mortality with chlorpyrifos methyl was lower (66\%) it was still much higher than for DDT (14\%) or pyrethroid (15\%) treatments. Nets impregnated with lambdacyhalothrin were compromized by resistance, killing only $30 \%$ of An. gambiae and $8 \%$ of $C x$. quinquefasciatus. Nets impregnated with chlorpyrifos methyl killed more (45\% of An gambiae and $15 \%$ of $C x$. quinquefasciatus), but its activity on netting was of short duration. Contact bioassays on the sprayed cement-sand walls over the nine months of monitoring showed no loss of activity of chlorpyrifos methyl, whereas lambdacyhalothrin and DDT lost activity within a few months of spraying.

Conclusion: As an IRS treatment against pyrethroid resistant mosquitoes chlorpyrifos methyl CS outperformed DDT and lambdacyhalothrin. In IRS campaigns, chlorpyrifos methyl CS should show higher, more-sustained levels of malaria transmission control than conventional formulations of DDT or pyrethroids. The remarkable residual activity indicates that cost-effective alternatives to DDT are feasible through modern formulation technology.

\section{Background}

International efforts to control malaria supported by the Global Fund and the President's Malaria Initiative (PMI) are underway in many parts of Africa using strategies

\footnotetext{
* Correspondence: raphael.n'guessan@lshtm.ac.uk

'Department of Infectious \& Tropical Diseases, London School of Hygiene and Tropical Medicine, Keppel Street, London, WC1E 7HT, UK
}

based on the scaling-up of long-lasting insecticidal nets (LLINs) and indoor residual spraying (IRS) [1-3]. Pyrethroids are the only group of insecticides currently recommended for use on mosquito nets [4]. Pyrethroid resistance has, in recent years, become widespread among anopheline mosquitoes in western and southern Africa and also occurs in eastern and central Africa 
[5-8]. The recent evolution and spread of pyrethroid resistance in West Africa in the Mopti (M) molecular form of Anopheles gambiae sensu stricto presents a grave threat to control because carriers of this particular resistance are not killed by pyrethroid-treated nets or residual spraying $[9,10]$. With the scaling-up of malaria control efforts the continued selection of pyrethroid resistance $[11,12]$ may compromise malaria control programmes and render this group of insecticides ineffective. It is important to investigate alternative insecticides on nets to supplement existing pyrethroid-based LLINs.

The target site insensitivity gene that confers knock down resistance $k d r$ to pyrethroids in $A n$. gambiae shows cross resistance to DDT [6]. Of the insecticides recommended by WHO for IRS, the most long-lasting and cost-effective is DDT [13]. No assessment of DDT has been made in areas where $k d r$ is prevalent. A smallscale trial of DDT is essential before any decision to redeploy DDT can be made in West Africa.

Because of DDT's damaging environmental impact, the Stockholm Convention on Persistent Organic Pollutants stipulates that 'countries are encouraged to reduce and eliminate the use of DDT over time and switch to alternative insecticides' [14]. The alternative classes of insecticide to DDT - the organophosphates, carbamates and pyrethroids - are more expensive and shorter-lived. For programmes that use DDT to remain viable it is necessary to develop long-lasting formulations of the alternative insecticides before DDT can be replaced [15]. Dow Agrosciences has among its portfolio of insecticides the organophosphate chlorpyrifos methyl which is effective against anophelines but applied as a wettable powder, the standard IRS formulation, it is too short-lived [16]. The company has, therefore, developed a microencapsulated formulation to improve residual activity. Its limited environmental persistence [16] and lack of cross resistance makes chlorpyrifos methyl a more attractive prospect than DDT for IRS should re-formulation increase its residual life. It also has the advantage of not selecting for pyrethroid resistance or undermining LLIN use.

This paper reports on an experimental hut trial in southern Benin of microencapsulated chlorpyrifos methyl. Efficacy is compared with that of DDT and the pyrethroid lambdacyalothrin in an area where An. gambiae has become difficult to control with pyrethroids [9].

\section{Methods}

\section{Study sites and experimental huts}

The evaluation was carried out in experimental huts situated in Ladji, a peri-urban village on the periphery of Cotonou, the capital of Benin. The village floods during the rainy season, creating breeding sites for $A n$. gambiae. The local An. gambiae population is comprised entirely of the $M$ taxon and is resistant to pyrethroids and DDT, with $k d r$ at high frequency and metabolic resistance also present $[9,17]$. The nuisance mosquito Culex quinquefasciatus is present year round and is resistant to pyrethroid, carbamate and organophosphate insecticides [17]. The experimental huts are made from concrete bricks, with roofs of corrugated iron, ceilings lined with plastic sheeting on the interior surface and walls plastered with a cement/sand mix [18-20]. Each hut stands on a concrete base surrounded by a water-filled moat to exclude scavenging ants. Entry of mosquitoes occurs via four slits, $1 \mathrm{~cm}$ wide, located on three sides of the hut. Mosquitoes exit into a verandah trap projecting from the fourth side.

\section{Insecticide treatments}

The insecticides used were:

- chlorpyrifos methyl 24\% CS ('Reldan GF 1246', Dow AgroSciences)

- DDT 50\% WP (sourced by Dow from South Africa)

- lambdacyhalothrin 2.5\% CS ('Icon', Syngenta, Switzerland) microencapsulation designed for ITNs.

- Lambdacyhalothrin 10\% WP ('Icon' Syngenta, Switzerland) wettable powder designed for IRS.

The following treatments and application rates were compared in seven experimental huts:

- chlorpyrifos methyl IRS at $500 \mathrm{mg} / \mathrm{m}^{2}$

- DDT IRS at $2 \mathrm{~g} / \mathrm{m}^{2}$

- Lambdacyhalothrin $10 \% \mathrm{WP}$, IRS at $30 \mathrm{mg} / \mathrm{m}^{2}$

- chlorpyrifos methyl ITN at $100 \mathrm{mg} / \mathrm{m}^{2}$

- lambdacyhalothrin $2.5 \% \mathrm{CS}$, ITN at $18 \mathrm{mg} / \mathrm{m}^{2}$

- unsprayed control hut

- untreated net

The Chlorpyrifos methyl dosage applied for IRS was recommended by Dow Agrosciences whereas the application rate for the ITN was the one used in experimental huts in Ivory Coast to control the Savanah (S) taxon of pyrethroid-resistant An. gambiae [21].

The dosages for DDT and lambdacyalothrin treatments are those conventionally used and recommended by WHO.

The test nets were made of 100-denier polyester netting in which a total of 80 holes of $4 \mathrm{~cm}^{2}$ area were cut along each side and end panel to simulate wear and tear. Nets were treated with insecticide by hand dipping. Insecticide was sprayed onto interior walls and plastic sheeting using a Hudson compression sprayer equipped with a flat fan nozzle. The evaluation started one week after treatment and ran for 42 nights from 8 April to 24 June 2005. 


\section{Study procedure}

The two net treatments and the untreated control net were rotated between three of the huts at six-day intervals. The four huts dedicated for IRS treatment were fixed throughout the study and the treatments could not, of course, be rotated. The volunteer sleepers gave informed consent and were provided with chemoprophylaxis. They slept in the huts from 20:00 to 05:00 each night, and were rotated between huts on successive nights to adjust for any variation in attractiveness to mosquitoes. Mosquitoes were collected each morning at 5:00 from floors, walls, ceilings and verandahs, and transported to the laboratory for identification to species, mortality counts and determination of gonotrophic condition. Live mosquitoes were held in plastic cups and delayed mortality was recorded after $24 \mathrm{~h}$. The effects of each treatment were expressed relative to the control in terms of:

- Deterrence: percentage reduction in the number of mosquitoes caught in treated hut relative to the number caught in the control hut;

- Induced exiting: percentage of the mosquitoes collected from the verandah trap of treated hut relative to percentage caught in verandah trap of control hut; - Inhibition of blood-feeding: percentage of the mosquitoes collected which was blood fed in the treated hut relative to percentage blood-fed in the control hut; - Induced mortality: percentage of dead mosquitoes in treated hut relative to percentage dead in control hut.

- If a treatment deters a significant number of mosquitoes from entering the hut, the values given by proportion blood feeding in the treatment hut may underestimate the full personal protective effect of the treatments. The personal protective effect of a treatment is better described by the reduction in the actual number of blood-fed mosquitoes in the treatment hut relative to the number blood-fed in the control hut:

$\%$ personal protection $=100\left(B_{u}-B_{t}\right) / B_{u}$

where $B_{u}=$ is the total number of blood-fed mosquitoes in the untreated control huts and $B_{t}$ is the total number blood-fed in the huts with insecticide treatment.

\section{Residual activity of insecticide treatments}

To assess residual activity on treated walls or nets cone bioassay tests were undertaken each month using 3-5 day old $A n$. gambiae females of a laboratory susceptible strain (Kisumu). Mosquitoes were exposed to nets for 3 min or to sprayed walls for $30 \mathrm{~min}$ as per WHO guidelines [22]. Approximately 50 mosquitoes were used per test.

\section{Data analysis}

Data were entered in Excel and transferred to STATA 6.0 software for further analysis. The numbers of mosquitoes collected each night and the actual numbers bloodfed was analysed per treatment using the KruskalWallis and Wilcoxon rank sum tests. Proportional data (exiting rate, blood-feeding, mortality) were analysed using logistic regression after adjusting for the effect of sleeper and hut. A Chi-square test for trend was performed to assess whether there was any change in mortality over time in bioassay tests.

\section{Results}

Trials of ITNs against An. gambiae and Cx. quinquefasciatus

Table 1 shows the total number of An. gambiae and $C x$. quinquefasciatus collected from the rooms with untreated or insecticide treated nets and the proportions exiting into the verandas. Figure $1 \mathrm{~A}$ shows the percentage blood-fed and dying among the total collected. An average of 14 An. gambiae and 19 Cx. quinquefasciatus females were collected each morning from the rooms and verandas of the huts with nets. There was no significant difference in the number of An. gambiae collected between the untreated control and chlorpyrifos methyl huts ( $\mathrm{p}=0.49$ ). By contrast, the number of An. gambiae found in the lambdacyhalothrin huts was $43.6 \%$ lower than in the untreated control huts $(\mathrm{P}<0.001)$. Hence, there was no evidence of deterrence with the organophosphate chlorpyrifos methyl in contrast to the pyrethroid lambdacyhalothrin ITN. The trends with $C x$. quinquefasciatus were similar to An. gambiae.

The chlorpyrifos-methyl- and lambdacyhalothrin-treated nets induced little or no additional exiting of $A n$. gambiae or $C x$. quinquefasciatus into the verandas over that recorded for untreated nets.

The proportions of $A n$. gambiae and Cx. quinquefasciatus blood-feeding through the sides or holes of the chlorpyrifos-methyl ITN were not significantly different from the untreated control nets or lambdacyhalothrin treated nets ( $\mathrm{P}>0.05$ for both species). However, the reduced entry rate of $A n$. gambiae to hut with lambdacyhalothrin-treated net indicated a $44.6 \%$ lower risk of exposure to potential infective bites than in huts with control or chlorpyrifos methyl-treated nets (table 1). Hence, there was no evidence of blood-feeding inhibition with chlorpyrifos-methyl- or lambdacyhalothrintreated nets though the pyretroid on net still procures 
Table 1 Summary results of mosquitoes hut frequenting habit and exit rates in huts.

\begin{tabular}{|c|c|c|c|c|c|}
\hline & Total entered & Average per night & Actual number fed & (\%) personnel protection & \% exiting $(\mathrm{Cl})$ \\
\hline \multicolumn{6}{|l|}{ ITNs } \\
\hline \multicolumn{6}{|l|}{ Anopheles gambiae s.s. } \\
\hline Untreated net & $689^{a}$ & 17.2 & 572 & - & $25.0^{\mathrm{a}}(21.7-28.2)$ \\
\hline$L C$ & $386^{b}$ & 9.7 & 317 & 44.6 & $29.0^{\mathrm{a}}(24.5-33.5)$ \\
\hline$C M$ & $648^{\mathrm{a}}$ & 16.2 & 518 & 9.4 & $34.2^{\mathrm{a}}(31.5-39.2)$ \\
\hline \multicolumn{6}{|l|}{ Culex quinquefasciatus } \\
\hline Untreated net & $845^{\mathrm{a}}$ & 21.1 & 531 & - & $29.8^{\mathrm{a}}(26.7-32.9)$ \\
\hline LC & $598^{\mathrm{b}}$ & 14.9 & 355 & 33.1 & $35.9^{\mathrm{a}}(32.1-39.8)$ \\
\hline CM & $839^{a}$ & 21.0 & 487 & 8.3 & $34.0^{\mathrm{a}}(30.6-37.1)$ \\
\hline
\end{tabular}

IRS

Anopheles. gambiae s.s.

\begin{tabular}{|c|c|c|c|c|c|}
\hline Unsprayed hut & $203^{\mathrm{a}}$ & 5.1 & 178 & - & $45.8^{\mathrm{a}}(39.8-52.7)$ \\
\hline LC & $117^{\mathrm{a}}$ & 2.9 & 86 & 51.7 & $58.1^{b}(49.2-67.1)$ \\
\hline CM & $420^{b}$ & 10.5 & 336 & 0 & $50.5^{\mathrm{a}}(43.4-58.5)$ \\
\hline DDT & $268^{\mathrm{a}}$ & 6.7 & 201 & 0 & $41.3^{\mathrm{a}}(36.0-47.7)$ \\
\hline
\end{tabular}

Culex quinquefasciatus

$\begin{array}{rll}\text { Unsprayed hut } & 858^{\mathrm{a}} & 21.4 \\ \text { LC } & 769^{\mathrm{a}} & 19.2 \\ \text { CM } & 817^{\mathrm{a}} & 20.4 \\ \text { DDT } & 764^{\mathrm{a}} & 19.1\end{array}$

$\begin{array}{ll}21.4 & 730 \\ 19.2 & 330 \\ 20.4 & 621 \\ 19.1 & 535\end{array}$

$\begin{array}{lcc}730 & - & 52.7^{\mathrm{a}}(49.3-56.0) \\ 330 & 54.8 & 54.6^{\mathrm{a}}(51.1-58.1) \\ 621 & 14.9 & 51.0^{\mathrm{a}}(47.6-55.2) \\ 535 & 26.7 & 49.7^{\mathrm{a}}(44.1-55.0)\end{array}$

Summary results of the impact of insecticide treated nets (ITNs) and indoor residual spray (IRS) treatments on hut frequenting habit, individual exposure to bites and exit rates of An. gambiae and CX. quinquefasciatus to the verandahs of the huts in Ladji. For each intervention arm (ITN and IRS) and mosquito species,

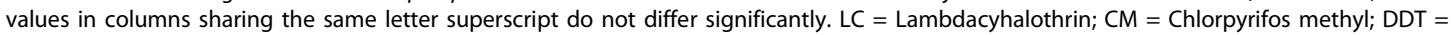

Dichlorodiphenyltrichloroethane

some appreciable degree of personal protection against pyrethroid-resistant An. gambiae or Cx. quinquefasciatus in this area of Benin.

The percentage mortality among An. gambiae was $45.2 \%$ with the chlorpyrifos methyl-treated net and only $29.8 \%$ with the lambacyalothrin-treated net (Figure 1B). Mortality rates among $C x$. quinquefasciatus were lower than among An. gambiae and did not exceed $15 \%$ with either type of treated net (Figure 1B).

Trials of IRS against An. gambiae and Cx. quinquefasciatus Table 1 shows the total number collected from the IRS treated rooms and the proportions exiting into the verandas. Figure $1 \mathrm{~B}$ shows the percentage blood-fed and dying among the An. gambiae and Cx. quinquefasciatus collections. Owing to differences in site attractiveness of individual huts, which were located at different places within the village, and to the inability to rotate IRS treatments, it was not possible to interpret the overall numbers collected from the rooms in terms of treatment effects. Differences in hut position were a confounding source of error. By serendipity a significantly larger number of $A n$. gambiae were collected from the chlorpyrifos-methyl treated hut than from the other types of hut (Table 1).
The percentages of An. gambiae and Cx. quinquefasciatus collected from the verandas of DDT, lambdacyhalothrin and chlorpyrifos methyl IRS treated huts were similar to those from the control verandas; the only exception was the significantly higher proportion of $A n$. gambiae, which exited the lambdacyhalothrin treated hut (Table 1).

Blood-feeding rates of An. gambiae and Cx. quinquefasciatus in the chlorpyrifos methyl and DDT treated huts were not significantly different from the untreated control (Figure 1A). In the lambdacyhalothrin treated hut half as many $C x$. quinquefasciatus were blood-fed compared to the control hut $(\mathrm{P}<0.001)$. However a similar trend was not observed with An. gambiae in the lambdacyhalothrin treated hut.

Mortality of pyrethroid resistant An. gambiae was 95.5\% with chlorpyrifos methyl IRS compared to 50.4\% in the hut sprayed with DDT and $30.8 \%$ in the hut sprayed with lambdacyhalothrin (Figure 1B). The mortality of $C x$. quinquefasciatus in the chlorpyrifos methyl IRS hut was $66.1 \%$ whereas in the DDT and lambdacyhalothrin IRS huts was only 14\% (Figure 1B). Chlorpyrifos methyl IRS showed greater potential than DDT or lambdacyhalothrin IRS for control of pyrethroid 


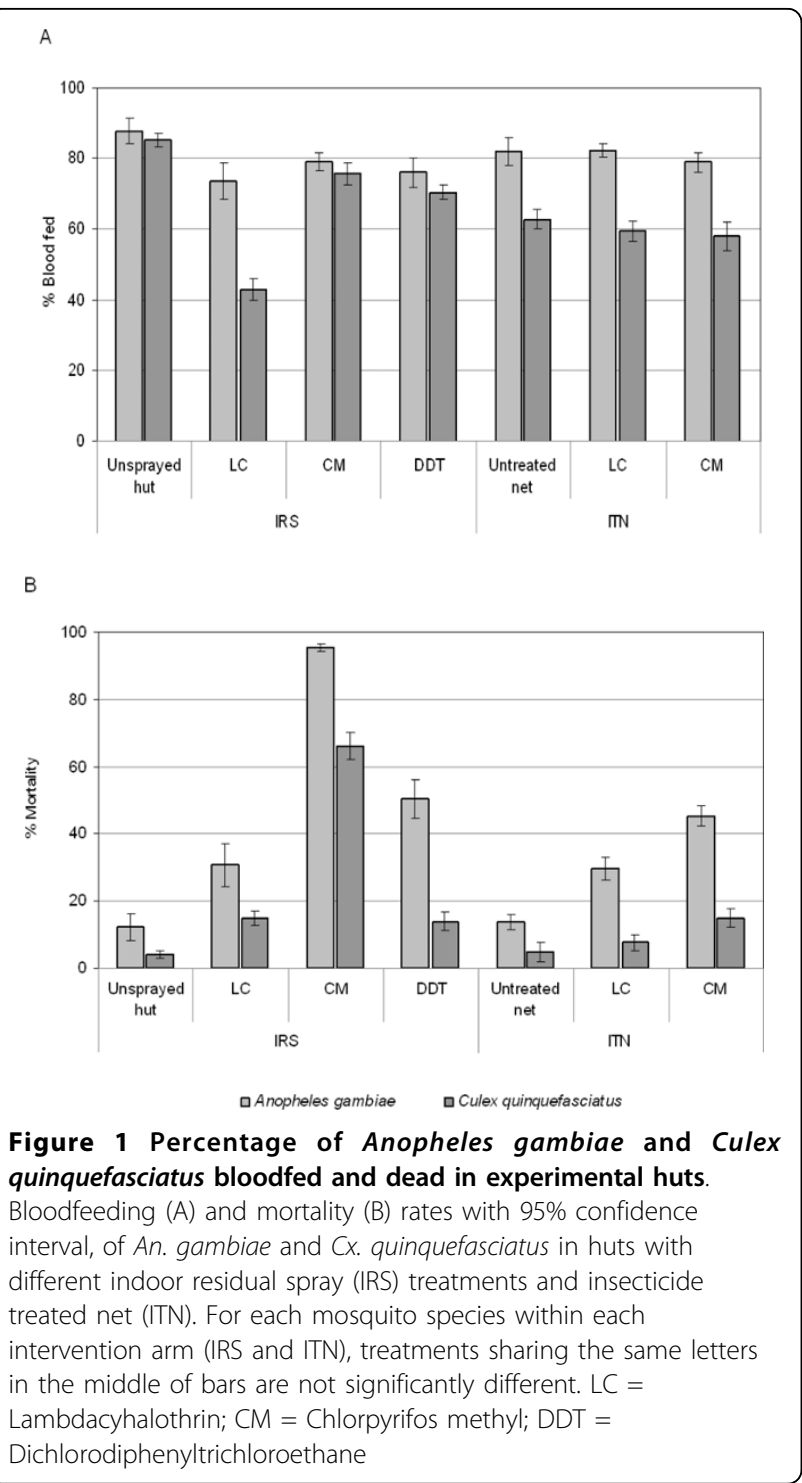

resistant An. gambiae $\mathrm{M}$ form and Cx. quinquefasciatus in areas of high $k d r$ frequency.

Neither chlorpyrifos methyl nor lambdacyhalothrin stained the sprayed surfaces nor did they cause an unpleasant odour or led to any complaints of adverse effects among the operators or sleepers at any stage of the study.

\section{Residual activity}

Figure 2 shows the mortality of An. gambiae freely entering the ITN and IRS treated huts divided into fortnightly intervals during the 42 days of the trial. The proportion of An gambiae killed in huts with chlorpyrifos methyl ITN during weeks $0-2$ was $73 \%$ after which mortality showed a progressive decline over the remaining six weeks $(\mathrm{P}<0.001)$ (Figure $2 \mathrm{~A})$. By contrast,

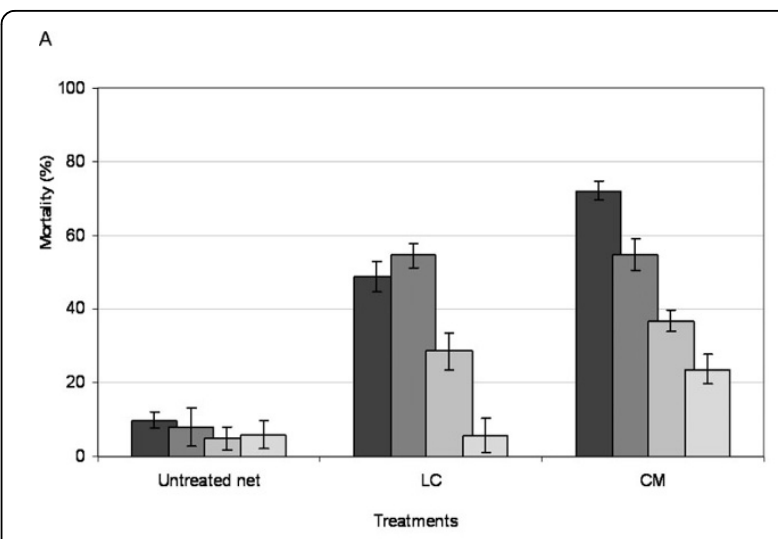

B

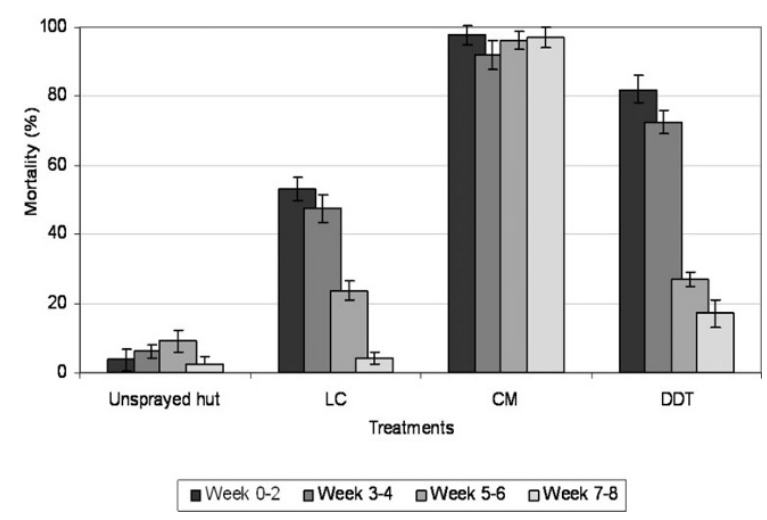

Figure 2 Mortality of Anopheles gambiae freely entering the ITN and IRS treated huts on a fortnightly basis. Anopheles gambiae mortality rates with $95 \%$ confidence interval, over fortnightly intervals during the hut trials with (A) insecticide treated nets (ITN) and (B) different indoor residual spray (IRS) treatments. Percentages are based on all mosquitoes collected from the rooms and veranda traps of huts. $\mathrm{LC}=$ Lambdacyhalothrin; $\mathrm{CM}=$ Chlorpyrifos methyl; DDT = Dichlorodiphenyltrichloroethane

chlorpyrifos methyl IRS consistently killed more than 95\% of pyrethroid resistant An. gambiae throughout the trial period. Mortality rates of An. gambiae in huts with DDT IRS and lambdacyhalothrin ITN or IRS decreased steadily between weeks $2-8(\mathrm{P}<0.0001)$, presumably due to loss of activity and survival of An. gambiae carriers of $k d r$ or other forms of pyrethroid resistance.

Figure 3 gives results of bioassay tests conducted with susceptible An. gambiae Kisumu strain on (A) ITNs and (B) IRS cement wall surfaces. The residual activity of chorpyrifos methyl on ITN confirmed the rapid decline in effectiveness, with the mortality rate decreasing from $100 \%$ to $9.7 \%$ within one just month $(\mathrm{P}<0.0001)$ (Figure 3A). Lambdacyhalothrin ITN remained highly active (100\% mortality) for up to 6 months before showing a decrease in mortality at month $9(\mathrm{P}=0.008)$.

The bioassays on chlorpyrifos methyl treated cement walls showed no loss of activity during the nine months 


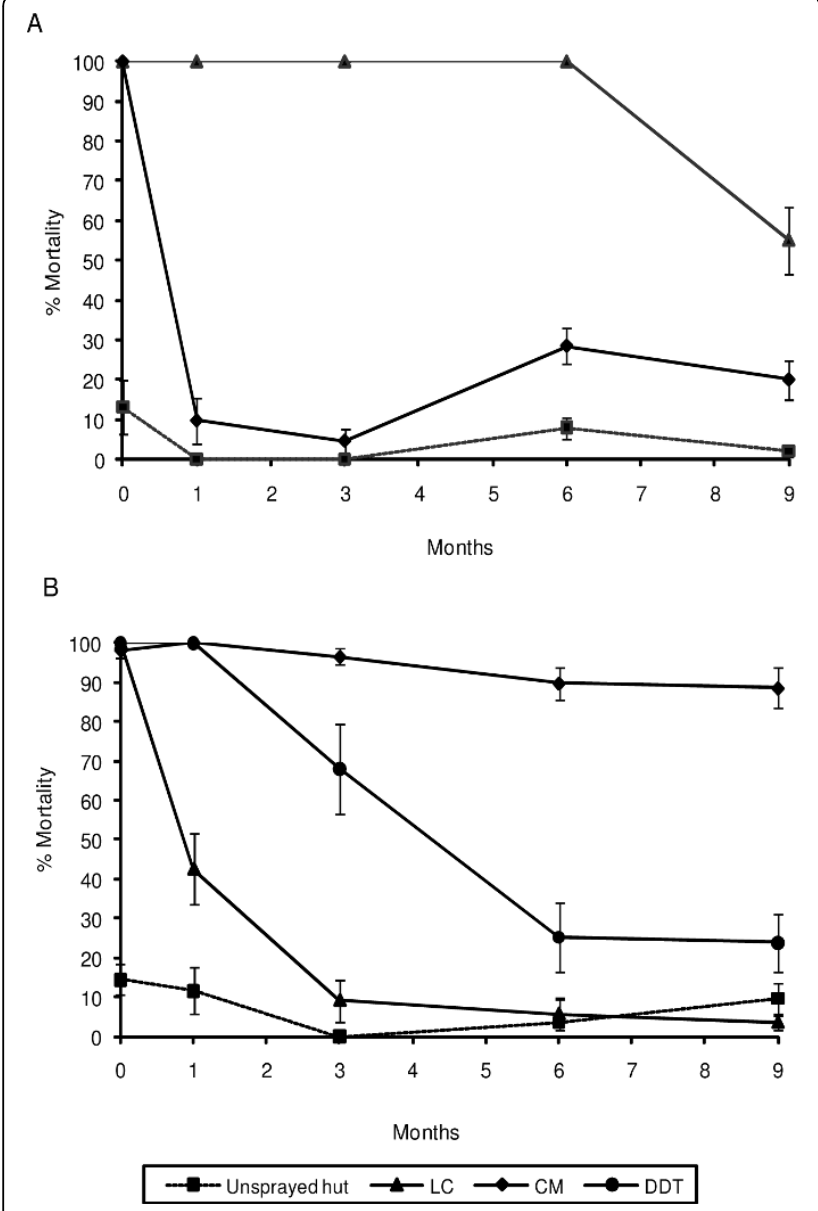

Figure 3 Bioassay tests monitoring the residual activity of treatments using the susceptible Anopheles gambiae Kisumu strain. Monitoring of the residual efficacy under WHO cone tests of (A) insecticide treated nets (ITN) and (B) different indoor residual spray (IRS) treatments against susceptible An. gambiae Kisumu strain in experimental huts at Ladji. LC = Lambdacyhalothrin; $C M=$ Chlorpyrifos methyl; DDT = Dichlorodiphenyltrichloroethane.

of follow-up ( $\mathrm{P}=0.79$ ) (Figure 3B). By contrast, decay of DDT and lambdacyhalothrin was evident on walls within the first month of spraying $(\mathrm{P}<0.001)$.

\section{Discussion}

Of the various groups of insecticide recommended by WHO for indoor residual spraying [23] the most costeffective, DDT, is compromised by its negative environmental impact, and the most widely used, the pyrethroids, will surely accelerate the selection of resistance and undermine that other great tool for malaria prevention, the long-lasting pyrethroid-treated net $[9,10,24]$. The ideal compound for IRS would come from an entirely different class of insecticide to pyrethroids or organochlorines. The formulations of organophosphates and carbamates currently recommended for IRS are relatively short-lived [23] and this had limited their deployment by malaria control programmes. In the present study, the microencapsulated chlorpyrifos methyl applied as IRS treatment in Southern Benin killed almost all pyrethroid and DDT resistant An. gambiae that entered the huts and demonstrated a residual activity that lasted for at least nine months without showing any decay detectable by bioassay. This constitutes an insecticide formulation to rival DDT and pyrethroids in terms of residuality and cost effectiveness.

Chlorpyrifos methyl CS has potential for deployment in a variety of epidemiological settings. In areas of West Africa where pyrethroids or DDT no longer control $A n$. gambiae because of resistance $[9,10]$ chlorpyrifos methyl CS makes a promising alternative. Indeed, use of chlorpyrifos methyl CS or other long lasting cholinesterase inhibitor will be essential to keep malaria control or malaria elimination targets on track. With national malaria burdens continuing to fall [24] epidemics may become more frequent and IRS, as an emergency response may become more widely practised. Chlorpyrifos methyl CS is a less risky choice than DDT or pyrethroid if the local resistance status of An gambiae is unknown, because organophosphate resistance in this species is comparatively rare compared to pyrethroid resistance [17].

With its remarkable efficacy and residual activity it is essential to consider the issue of organophosphate resistance management from the outset. The most pragmatic approach to manage insecticide resistance is to rotate insecticides with differing modes of action between spray campaigns, although in reality sequential substitution of one unrelated compound for another once the former has failed is more the norm [25]. Ideally the use of pyrethroids should be constrained in order to preserve ITNs and LLINs. A promising partner insecticide to rotate with chlorpyrifos methyl in spray programmes is chlorfenapyr as this novel insecticide shows no cross resistance to OPs or pyrethroids in An. gambiae or $C x$. quinquefasciatus, and has shown potential for IRS in areas where resistance to these two classes of insecticide has not yet been reported [26].

The short-lived residual activity of DDT in this trial ( $<2$ months) compared to conventional wisdom $(>6$ months) might be due to the formulation obtained, to the cement substrate on which this insecticide was applied or to its residual activity perhaps being over stated [23]. Longer residual activity of DDT has been observed elsewhere on wooden walls in villages in Brasil [27] or Madagascar [28], but wood substrates are known to be rather benign to all types of insecticide. The study conducted in Southern Benin provides no reassurance that the activity shown by DDT would provide effective 
or sustainable control of malaria in areas where pyrethroid resistance involving $k d r$ has become the norm.

There is a deterrent effect of lambdacyhalothrin-treated net against pyrethroid-resistant An. gambiae in Southern Benin and trustworthy higher personal protection than chlorpyrifos methyl because treatment could be rotated. For the lambdacyhalothrin IRS the result is less trustworthy because IRS treatments could not be rotated and the huts showed site effects with respect to mosquito numbers.

The hut trial also demonstrates the potential of chlorpyrifos methyl on nets for control of pyrethroid-resistant vectors. During the first few weeks, before chlorpyrifos methyl started to decay or wear off, the level of mortality was $100 \%$ against An. gambiae. With the advent of LLIN treatment technology there is scope for improving residual efficacy on nets using appropriate binding or incorporation technology.

\section{Conclusion}

With its good safety profile, low mammalian toxicity and residual activity, chlorpyrifos methyl meets the profile of a cost-effective replacement for DDT or pyrethroids. The challenge of finding an environmentally acceptable alternative to DDT appears to be met. Adopting a IRS strategy that incorporates chlorpyrifos methyl will reduce the selective pressure generated by pyrethroids and help preserve the future of LLINs.

Despite the great promise shown by chlorpyrifos methyl it seems unlikely that the manufacturer, Dow AgroSciences, will deploy the long-lasting formulation in the near future, because of uncertainties in the vector control market. The comparatively small size of the market and the unpredictability of winning tenders is a major deterrent to companies not already engaged in malaria control. The manufacturer should be encouraged by international donors and technical authorities to pursue further development and evaluation against malaria indicators in endemic settings where An. gambiae is pyrethroid-resistant or IRS is being considered for malaria control.

\section{Acknowledgements}

We wish to thank the volunteer residents of Ladji for participating in the trial and to Dr Driss Kelili of Dow Agrosciences for his encouragement and provision of test products. This work was funded by a grant from the Gates Malaria Partnership to the London School of Hygiene and Tropical Medicine. The study received approval from the Ministry of Health, Cotonou, Republic of Benin, on 30 March 2005 (approval no. 10715/MSP/DG/SGM/DRS).

\section{Author details}

'Department of Infectious \& Tropical Diseases, London School of Hygiene and Tropical Medicine, Keppel Street, London, WC1E 7HT, UK. ${ }^{2}$ CREC laboratories, Centre de Recherche Entomologique de Cotonou, Laboratoire Nationale, Ministère de la Santé, Cotonou 06 BP 2604, Benin.

\section{Authors' contributions}

RN co-designed and supervised the project, data analysis, drafted the manuscript.

$\mathrm{PB}, \mathrm{AO}$ and $\mathrm{JC}$ conducted the field trials and bioassay testing.

MA Director of Centre de Recherche Entomologique de Cotonou,

supervision and facilitation, reviewed and revised the manuscript.

MR coordination with $\mathrm{WHO}$ and manufacturers, co-designed the project,

data interpretation, revised the manuscript.

All authors read and approved the final manuscript.

\section{Authors' information}

Raphael N'Guessan, Martin Akogbeto and Mark Rowland are all associated with the Pan African Malaria Vector Research Consortium, http://www. pamverc.org.

\section{Competing interests}

The authors declare that they have no competing interests.

Received: 26 October 2009

Accepted: 8 February 2010 Published: 8 February 2010

\section{References}

1. Bhattarai A, Ali SS, Kachur SP, Martesson A, Abbas AK, Khatib R, AlMafazy AW, Ramsan M, Rotllant G, Gerstenmaier JF, Molteni F, Adulla S, Montgomery SM, Kaneko A, Bjorkman A: Impact of artemisinin-based combination therapy and insecticide-treated nets on malaria burden in Zanzibar. PLoS Medicine 2007, 4:309.

2. Okiro EA, Hay SI, Gikandi PW, Sharif SK, Noor AM, Peshu N, Marsh K, Snow RW: The decline in paediatric malaria admissions on the coast of Kenya. Malar J 2007, 15:151.

3. Ceesay SJ, Casals-Pascual C, Erskine J, Anya SE, Duah NO, Fulford AJC, Sesay SSS, Abubakar I, Dunyo S, Sey O, Palmer A, Fofana M, Corrah T, Bojang KA, Whittle HC, Greenwood BM, Conway DJ: Changes in malaria indices between 1999 and 2007 in The Gambia: a retrospective analysis. Lancet 2008, 372:1545-1554.

4. Zaim M, Aitio A, Nakashima N: Safety of pyrethroid-treated mosquito nets. Med Vet Entomol 2000, 14:1-5.

5. Elissa N, Mouchet J, Riviere F, Meunier JY, Yao K: Resistance of Anopheles gambiae s.s. to pyrethroids in Côte d'Ivoire. Ann Soc Belg Med Trop 1993, 73:291-294.

6. Chandre F, Darriet F, Manga L, Akogbeto M, Faye O, Mouchet J, Guillet P: Status of pyrethroid resistance in Anopheles gambiae sensu lato. Bull World Health Organ 1999, 77:230-234.

7. Ranson H, Jensen B, Vulule JM, Wang X, Hemingway J, Collins FH: Identification of a point mutation in the voltage-gated sodium channel gene of Kenyan Anopheles gambiae associated with resistance to DDT and pyrethroids. Insect Mol Biol 2000, 9:491-497.

8. Hargreaves K, Koekemoer LL, Brooke BD, Hunt RH, Mthembu J, Coetzee M: Anopheles funestus resistant to pyrethroid insecticides in South Africa. Med Vet Entomol 2000, 14:181-189.

9. N'Guessan R, Corbel V, Akogbéto M, Rowland M: Reduced efficacy of insecticide-treated nets and indoor residual spraying for malaria control in pyrethroid resistance area, Benin. Emerg Infect Dis 2007, 13:199-206.

10. Sharp BL, Ridl FC, Govender D, Kuklinski J, Kleinschmidt I: Malaria vector control by indoor residual insecticide spraying on the tropical island of Bioko, Equatorial Guinea. Malar J 2007, 6:52.

11. Protopopoff N, Verhaeghen K, Van Bortel W, Roelants P, Marcotty T, Baza D, D'Alessandro U, Coosemans M: A significant increase in kdr in Anopheles gambiae is associated with an intensive vector control intervention in Burundi highlands. Trop Med Int Health 2008, 13:1479-1487.

12. Czeher C, Labbo R, Arzika I, Duchemin JB: Evidence of increasing Leu-Phe knockdown resistance mutation in Anopheles gambiae from Niger following a nationwide long-lasting insecticide-treated nets implementation. Malar J 2008, 7:189.

13. WHO: Indoor residual spraying: use of indoor residual spraying for scaling up global malaria control and elimination. Geneva 2006http:// malaria.who.int/docs/IRS-position.pdf.

14. United Nations Environment Programme: Stockholm Convention on Persistant Organic Pollutants (POPs). UNEP/Chemicals/2001 2001, 3-50.

15. WHO: Action plan for the reduction of reliance on DDT in disease vector control. WHO/SDENSH/015 2001. 
16. Tomlin CDS: The pesticide manual, A world compendium. British Crop Protection Council, London, UK, 122000.

17. Corbel V, N'Guessan R, Brengues C, Chandre F, Djogbenou L, Martin T, Akogbéto M, Hougard JM, Rowland M: Multiple insecticide resistance mechanisms in Anopheles gambiae and Culex quinquefasciatus from Benin, West Africa. Acta Trop 2007, 101:207-216.

18. Corbel V, Chandre F, Brengues C, Akogbeto M, Lardeux F, Hougard JM, Guillet P: Dosage-dependent effects of permethrin-treated nets on the behaviour of Anopheles gambiae and the selection of pyrethroid resistance. Malar J 2004, 3:22.

19. Darriet F, N'Guessan R, Koffi AA, Konan L, Doannio JMC, Chandre F, Carnevale P: [Impact of pyrethrin resistance on the efficacity of impregnated mosquito nets in the prevention of malaria: results of tests in experimental cases with deltamethrin SC. Bull Soc Pathol Exot 2000, 93:131-134.

20. N'Guessan R, Darriet F, Doannio JMC, Chandre F, Carnevale P: Olyset Net efficacy against pyrethroid-resistant Anopheles gambiae and Culex quinquefasciatus after 3 years' field use in Côte d'lvoire. Med Vet Entomol 2001, 15:97-104.

21. Asidi AN, N'Guessan R, Koffi AA, Curtis CF, Hougard JM, Chandre F, Corbel V, Darriet F, Zaim M, Rowland MW: Experimental hut evaluation of bednets treated with an organophosphate (chlorpyrifos-methyl) or a pyrethroid (lambdacyhalothrin) alone and in combination against insecticide-resistant Anopheles gambiae and Culex quinquefasciatus mosquitoes. Malar J 2005, 4:25.

22. WHO: Test procedures for insecticide resistance monitoring in malaria vectors, bio-efficacy and persistence of insecticides on treated surfaces. WHO/CDS/MAL/98.12, Geneva, Switzerland .

23. WHO: Pesticides and their application for the control of vectors and pests of public health importance. WHO/CDS/NTD/WHOPES/GCDPP/2006.1 Sixth 2006.

24. Feachem RGA, Phillips AA, Targett GA: Shrinking the Malaria Map: a prospectus on malaria elimination. Global Health Sciences, University of California, San Francisco 2009.

25. Raghavendra K, Subbarao SK: Chemical insecticides in malaria vector control in India. ICMR Bulletin 2003, 32(10).

26. N'Guessan R, Boko P, Odjo A, Knols B, Akogbeto M, Rowland M: Control of pyrethroid-resistant Anopheles gambiae and Culex quinquefasciatus mosquitoes with chlorfenapyr in Benin. Trop Med Int Health 2009, 14:389-395.

27. Charlwood JD, Alecrim WD, Fe N, Mangabeira J, Martins VJ: A field trial with Lambda-cyhalothrin (ICON) for the intradomiciliary control of malaria transmitted by Anopheles darlingi Root in Rondonia, Brazil. Acto Trop 1995, 60:3-13.

28. Brutus L, Le Goff G, Rasoloniaina LG, Rajaonarivelo V, Raveloson A, Cot M: The campaign against malaria in central western Madagascar: comparison of the efficacy of lambda-cyhalothrin and DDT house spraying. I-Entomological study. Parasite 2001, 8:297-308.

doi:10.1186/1475-2875-9-44

Cite this article as: N'Guessan et al:: Control of pyrethroid and DDTresistant Anopheles gambiae by application of indoor residual spraying or mosquito nets treated with a long-lasting organophosphate insecticide, chlorpyrifos-methyl. Malaria Journal 2010 9:44.

\section{Submit your next manuscript to BioMed Central and take full advantage of:}

- Convenient online submission

- Thorough peer review

- No space constraints or color figure charges

- Immediate publication on acceptance

- Inclusion in PubMed, CAS, Scopus and Google Scholar

- Research which is freely available for redistribution

Submit your manuscript at www.biomedcentral.com/submit 